\title{
Long non-coding RNAs are major contributors to transcriptome changes in sunflower meiocytes with different recombination rates
}

\author{
Nathalia M. V. Flórez-Zapata ${ }^{1}$, M. Humberto Reyes-Valdés² and Octavio Martínez ${ }^{1 *}$
}

\begin{abstract}
Background: Meiosis is a form of specialized cell division that marks the transition from diploid meiocyte to haploid gamete, and provides an opportunity for genetic reassortment through recombination. Experimental data indicates that, relative to their wild ancestors, cultivated sunflower varieties show a higher recombination rate during meiosis. To better understand the molecular basis for this difference, we compared gene expression in male sunflower meiocytes in prophase I isolated from a domesticated line, a wild relative, and a F1 hybrid of the two.

Results: Of the genes that showed differential expression between the wild and domesticated genotypes, $63.62 \%$ could not be identified as protein-coding genes, and of these genes, $70.98 \%$ passed stringent filters to be classified as long non-coding RNAs (IncRNAs). Compared to the sunflower somatic transcriptome, meiocytes express a higher proportion of IncRNAs, and the majority of genes with exclusive expression in meiocytes were IncRNAs. Around $40 \%$ of the IncRNAs showed sequence similarity with small RNAs (sRNA), while $1.53 \%$ were predicted to be sunflower natural antisense transcripts (NATs), and $9.18 \%$ contained transposable elements (TE). We identified 6895 IncRNAs that are exclusively expressed in meiocytes, these IncRNAs appear to have higher conservation, a greater degree of differential expression, a higher proportion of sRNA similarity, and higher TE content relative to IncRNAs that are also expressed in the somatic transcriptome.
\end{abstract}

Conclusions: IncRNAs play important roles in plant meiosis and may participate in chromatin modification processes, although other regulatory functions cannot be excluded. IncRNAs could also be related to the different recombination rates seen for domesticated and wild sunflowers.

Keywords: Meiosis, Prophase I, Homologous recombination, IncRNA, miRNA, RNA-seq, Transcriptomics

\section{Background}

Meiosis is a complex cell division process that generates haploid gametes. During prophase I, the first and longest meiotic stage [1], chromosomes pair, synapse and recombine [2], which promotes increased genetic variation [3] and proper chromosome segregation in subsequent stages [4]. As expected, these meiotic events must be

\footnotetext{
* Correspondence: omartine@langebio.cinvestav.mx; octavio.martinez@cinvestav.mx

'Laboratorio Nacional de Genómica para la Biodiversidad (LANGEBIO)/Unidad de Genómica Avanzada, Centro de Investigación y de Estudios Avanzados del Instituto Politécnico Nacional (Cinvestav), 36821 Irapuato, Guanajuato, México

Full list of author information is available at the end of the article
}

tightly coordinated. In yeast, the presence of transcriptional regulatory elements and temporary changes in gene expression highlight how transcriptional regulation may contribute to this coordination [5-7]. Several plants encode the transcriptional regulator gene $M M D 1[8,9]$, while other genes such as the LISCL gene in lily and AMEIOTIC1 in maize are putative transcriptional regulators [10-12]. However, the transcriptional regulatory mechanisms involved in plant meiosis remain poorly understood $[9,13]$.

Advances in sequencing technologies and meiocyte collection techniques enabled the generation of transcriptomes for pure meiocytes in Arabidopsis [14, 15], 
maize [16], and sunflower [17]. These studies allowed the identification of new transcriptional regulatory elements $[18,19]$ and meiotic genes [20], and raise new questions about the transcriptional behavior of meiotic cells, particularly given the high levels of expression of transposable elements (TEs) in Arabidopsis meiocytes and mitochondrial genes in maize and Arabidopsis [14-16].

Additionally, the high transcriptional activity of meiocytes is remarkable. Around 20,000 genes in Arabidopsis and 30,000 in maize and sunflower are expressed [14-17]; these figures are comparable with the number of genes expressed in seedlings (which contain different tissues and cell types), suggesting that transcription during meiosis may be very promiscuous [13]. Nevertheless, many genes expressed in meiocytes correspond to unannotated features in the genome [19] or transcripts without a protein coding ortholog [17]. Some of these unannotated transcripts could be non-coding RNAs, especially since non-protein coding transcripts reportedly represent the majority of transcribed genes in eukaryote transcriptomes [21]. Non-coding RNAs (ncRNAs) are a diverse group of transcripts that includes housekeeping RNAs (e.g. ribosomal RNA, transfer RNA) and regulatory ncRNA [22]. Within the ncRNA regulatory group are long non-coding RNAs (lncRNA), which are $>200 \mathrm{nt}$ transcripts that do not encode a protein and can act as cis- or trans-regulators of gene transcription or as protein scaffolds in chromatin-modifying complexes [21, 23-25]. On the other hand, small RNAs (sRNA) are 20-27 nucleotide (nt) regulatory ncRNA that participate in post-transcriptional gene regulation and genome stability maintenance $[26,27]$. Recently, sRNA and IncRNA were associated with the regulation of plant meiosis and fertility, although their specific function awaits clarification [28-31].

We previously showed that sunflower (Helianthus annus L.) is a good model for studying plant meiosis [17] because its inflorescence contains a large number of disk flowers that have different ages (growing older with the progression from the head center to the periphery) [32], which allows the isolation of nearly pure populations of male meiocytes in well-defined meiotic stages. In this study we sequenced the transcriptome of prophase I meiocytes from three different sunflower genotypes that were previously found to have significantly different recombination rates, inferred from chromosome pairing index in [33]. Interestingly, the largest proportion ( $64 \%)$ of differentially expressed genes (DEG) were not protein coding genes, but passed stringent filters to be classified as lncRNA. These lncRNAs are highly meiosis-specific and although some have sRNAassociated functions, others showed no connection with sRNA-mediated regulation, suggesting that lcRNAs may participate in other regulatory mechanisms. We propose that lncRNAs play a protagonist role in regulating meiotic gene expression or chromatin state changes during meiosis, which could also be related to the observed differences in the homologous recombination rate of the sunflower genotypes that we studied, as well as to other possible domestication-related meiotic traits.

\section{Results and discussion}

Differentially expressed genes between male sunflower meiocytes with different recombination rates

Chromosome pairing was significantly higher in a domesticated sunflower genotype (elite line HA89) relative to a wild sunflower genotype (Ac-8). Meanwhile, an F1 (F1) hybrid resulting from intercrossing these two genotypes had an intermediate rate of chiasmate chromosome arms [33]. The effect of domestication on the recombination rate was previously documented by RossIbarra [34], who observed that domesticated plants have higher recombination rates than their wild relatives, and proposed that domestication selects for this increased recombination. As a first approach to understand which genes or regulatory processes could be related to variations in chiasma frequency, we conducted a transcriptome analysis of sunflower prophase I meiocytes from Ac-8 (wild type, $H$. annuus ssp. texanus), HA89 (domesticated elite line, $H$. annuus var. macrocarpus), and an F1 hybrid of the two, which correspond to the genetic materials used in the previous report of comparative analysis of chiasmate chromosome arms [33].

We obtained $\sim 8.6 \times 10^{8}$ pair-end reads for the sunflower meiocytes (see Methods and Table AF1-1 in Additional file 1). The F1 genotype reads were qualitytrimmed and used for a de novo transcriptome assembly, which was used as a reference transcriptome for subsequent analyses (see section "Sequencing and assembly results" in Additional file 1). Around $78 \%$ of the reads (Table AF1-1 in Additional file 1) mapped to a unique position within one of the 73,658 distinct transcripts ("genes") in this transcriptome. More than half the transcripts $(39,354,53.42 \%)$ were annotated via BLAST with any of the peptide databases queried (see Methods), while the remaining 34,304 transcripts (46.58\%) could not be identified using this approach. Identified transcripts that shared the same BLAST identifier were considered to be either products of the same sunflower locus or derived from closely related paralogs. To quantify the expression of these transcripts, reads aligned to these loci were added and the transcripts were "collapsed" to treat the related transcripts as a single gene. The final dataset included 59,085 genes.

To estimate how many genes could be missing from our sample, we applied the method described by GarcíaOrtega and Martínez [35]. The estimate for the number of missing genes in our dataset was equal to zero, and 
the $95 \%$ confidence interval for the number of missing genes was zero to three, indicating that, within the total sample, our RNA-seq experiment detected practically all expressed transcripts and thus no extra sample was needed to detect missing genes. This result implies that genes detected only in meiocytes and not in somatic tissues are likely to have exclusive meiocyte expression.

We found 29,469 (49.87\%) differentially expressed genes (DEGs) between the domesticated and wild genotype using a False Discovery Rate (FDR) of $1 \%$. The majority (63.62 \%) of these genes could not be identified via BLAST (Fig. 1), which is consistent with our previous study wherein transcripts that could not be identified were more abundant in meiocytes than in the somatic transcriptome, and also exhibited more tissue-specific expression [17]. Thus, we focused on these unidentified transcripts to investigate how many could be classified as lncRNAs that may play a regulatory role in homologous recombination during meiosis.

\section{IncRNA identification in sunflower meiocytes}

To test if the unidentified transcripts expressed in sunflower meiocytes were lncRNAs, we used the workflow described in Fig. 2. First, we excluded all 39,354 transcripts with protein coding potential using BLAST with protein databases (see Methods). For the remaining 34,304 unknown transcripts we performed a BLASTN search against the draft genome sequence compiled by the Sunflower Genome Project (in progress for the inbred line HA412). Among these transcripts, $90.88 \%$ had a BLAST hit that was more than $90 \%$ identical to the genome draft. This result suggests that most of these sequences were indeed sunflower transcripts and not assembly artifacts. On the other hand, discarding the transcripts that lacked a genome hit would be unwise, as they may be products of RNA processing or genotype-specific sequences. Thus, we tested both unknown transcripts (with and without genome hit), for their protein coding potential with two different algorithms: CPC (Coding Protein Calculator) [36] and CPAT (Coding-Potential Assessment Tool) [37], and only those transcripts that passed the thresholds of both algorithms were classified as lncRNAs (See Methods). Given that the methods used by CPC and CPAT are complementary (CPC uses a support vector machine classifier, while CPAT employs a logistic regression model), the classification of sequences as IncRNA only when both algorithms concurred can be considered highly trustworthy (see "Additional discussion of lncRNA Identification" in Additional file 1).

Of the unknown transcripts with and without a genome hit, $74.17 \%(23,960)$ and $68.28 \%$ (1367), respectively, were classified as lncRNAs (Fig. 2). Other transcripts that did not pass one or both of the coding potential filters (CPC and CPAT) were designated as unclassified. We examined the expression of the lncRNAclassified transcripts in the three sunflower genotypes, and found that $75.65 \%(18,128)$ of the lncRNAs with a genome hit showed evidence of expression in all three sunflower genotypes tested, so these can be considered as highly reliable sunflower lncRNAs (Fig. 2a). On the other hand, $32.26 \%$ (441) of the IncRNAs without a genome hit showed expression in all three sunflower genotypes (Fig. 2b).

In contrast, the proportion of IncRNA with a genomic hit detected in the F1 and wild genotypes was only $11.03 \%$ (2644 of 23,960$)$, while those without a genomic hit represented approximately $53.62 \%$ (733 of 1367) of the total. This enrichment in the 'wild origin' lncRNA without a genome hit may be to due to our use of a domesticated genotype (inbred line HA412) as a reference.

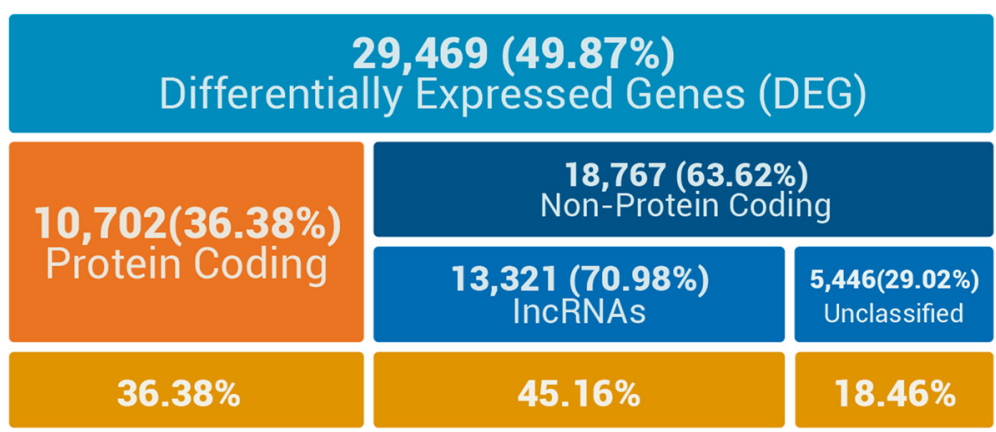

Fig. 1 Diagram showing the numbers and proportions of differentially expressed genes (DEG) between the domesticated and wild genotypes grouped by coding class. Global percentage of DEGs (first row) was calculated based on the total number of genes $(59,058)$. Percentages of genes with and without protein coding potential were calculated with reference to number of DEGs $(29,469)$. Percentages of IncRNA and unclassified genes were calculated with reference to non-protein coding genes $(18,767)$. Last row in the diagram presents percentages in each category with reference to the total DEG number $(29,469)$ 


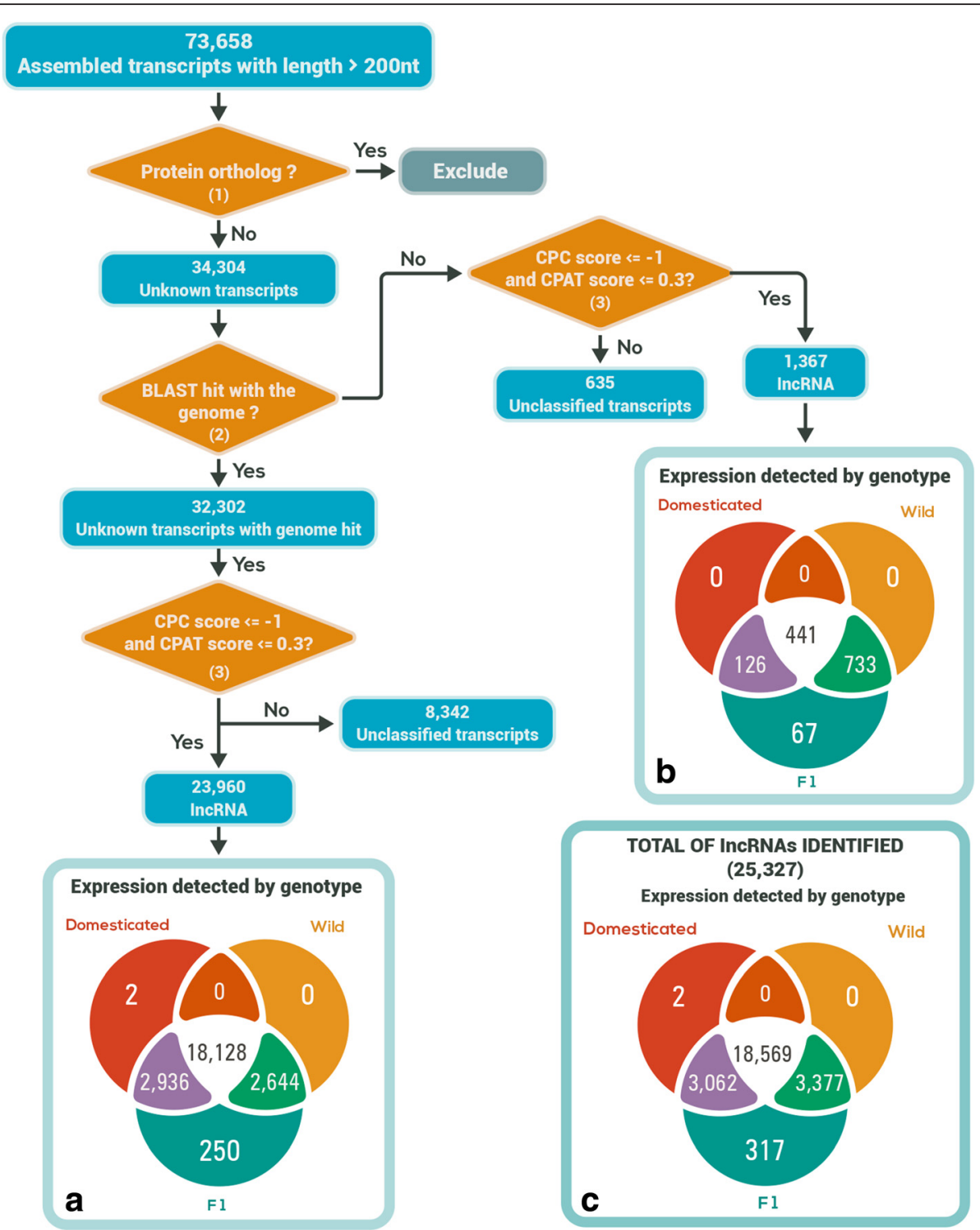

Fig. 2 Bioinformatic pipeline to determine the IncRNA nature of transcripts and Venn diagrams with number of IncRNA expressed by genotype. Protein coding was determined by comparing transcripts with peptide databases (TAIR 10, NCBI RefSeq and sunflower peptides); all transcript with one or more hits to peptides with a bitscore $\geq 90$ and $E$ value $<1$ e- 6 were discarded as potential IncRNAs in (1). To determine if the transcripts had a blast hit with the draft of the sunflower genome, a threshold of bitscore $\geq 90$ and E-value $<1$ e- 6 was employed in (2). Only transcripts with a CPC score $\leq-1$ and CPAT score $\leq 0.3$ were considered as IncRNA by filters in (3). Venn diagrams: a) Expression of IncRNAs with significant similarity with the sunflower genome. $\mathbf{b}$ Expression of IncRNAs with no significant similarity with the sunflower genome. $\mathbf{c}$ Total number of IncRNAs detected

A Venn diagram of all lncRNAs showed that all but two (>99.99 \%) were detected in the F1 hybrid, and the remaining two lncRNAs appeared only in the domesticated genotype (Fig. 2c). The majority (73.31 \%; 18,569) of the IncRNAs were detected in the F1 and both parental genotypes (inner intersection), while similar proportions were detected in the F1 and one of the parental genotypes (12.08 \% (3062) and $13.33 \%$ (3377) for F1domesticated and F1-wild, respectively). Interestingly, $317(1.25 \%)$ of the lncRNAs were exclusively detected in the F1. This expression pattern suggests that processing of the original transcript to a mature lncRNA could be affected by the interaction between the wild and cultivated genomes. After transcription, most lncRNAs are processed similarly to protein-coding RNAs, including 5 '-end capping, 3 '-end polyadenylation, and splicing modifications [38]. Although alternative splicing appears to be less common in plants than in animals, in grape plants expression of alternative spliced forms is reportedly genotype-dependent [39]. 
Among the 25,327 IncRNAs identified, 13,321 showed differential expression between the domesticated and wild genotypes. qRT-PCR analysis of a selected set of differentially expressed lnc-RNAs was performed. For the five lncRNAs where the qRT-PCR were completed, the tendencies in fold change between the domesticated and wild genotypes were validated; see section "qRTPCR analysis of selected lncRNAs" in Additional file 1 for details. The proportion of DEG lncRNAs and unclassified transcripts was significantly higher than the proportion of protein-coding DEGs $(45.16 \%$ and $36.38 \%$, respectively, $P<0.01$ ) (Fig. 1). This result indicates that the major changes observed at the transcriptomic level between meiocytes of domesticated and wild origin are due to changes in IncRNA gene expression, which could be related to the differential recombination rates between these genotypes. A recent study by Ding et al. [40] highlighted the importance of lncRNAs in fission yeast meiosis by demonstrating that the sme 2 gene that encodes a meiosis-specific lncRNA is important for homologous recognition and homologous chromosome pairing. Although no meiosis-specific lncRNA are yet known in plants [13], many lncRNAs are associated with sexual reproduction and fertility. For example, the maize lncRNA $Z m 401$ is thought to be essential for tapetum and microspore development [41], while rice LDMAR IncRNA regulates photoperiod-sensitive male sterility and normal pollen development [42]. Indeed, a large number of lncRNAs important for sexual reproduction in rice have been identified through genome wide screening [29].

Transcriptome analysis of mammalian testes showed that the gene expression levels in this organ are higher relative to other organs (e.g., brain, heart, liver, kidney), and this difference is more pronounced for predicted lncRNAs, which have higher expression in testis than in other organs [43]. Concordantly, we previously demonstrated that in humans the testis has the highest transcriptome diversity [44]. Moreover, the repertoire and expression pattern of lncRNAs in tetrapods showed that lncRNAs are preferentially expressed in the testes, and this expression is actively regulated, which suggests that this expression is not due only to non-specific transcription in open chromatin regions [45]. The co-expression networks of lncRNAs with protein-coding genes showed that the clusters with the highest lncRNA proportions were enriched in spermatogenesis functions, which is in agreement with the high proportions of lncRNAs in the testes, as well as the substantial contribution that pachytene spermatocytes make to the transcriptome of whole testes [43, 45]. On the other hand, a genome-wide characterization of maize lncRNAs showed that male reproductive tissues such as immature tassels, anthers, and pollen, had higher lncRNA levels than did other tissues
[46]. Thus, this lncRNA enrichment in animal and plant reproductive tissues during meiosis and gametogenesis may arise from a conserved and well-structured regulation of gene expression that involves lncRNAs.

The molecular mechanisms by which lncRNAs could participate in plant meiosis are not well understood. According to their function in other biological processes, they may play roles in controlling gene expression, influencing epigenetic factors, maintaining characteristics of heterochromatin, or controlling transposable elements [13]. In rice, more than 700 lncRNAs appear to be key factors for inducing the biogenesis of $21 \mathrm{nt}$ phased siRNAs (phasiRNAs) that are associated with the germline-specific MEL1 argonaute protein [47], implying that in meiosis lncRNAs could also act as precursors or mimics of sRNA targets [48]. In addition, lncRNAs could play a direct structural role similar to the human skin fibroblast cell line lncRNA DDSR1, which interacts with BRCA2 to modulate DNA repair by homologous recombination [49], a repair pathway that is also important for proper meiosis in Arabidopsis [50].

Differences in lncRNA expression among genotypes and consequent phenotypic effects of these differences have been observed in wheat, wherein the expression of two lncRNAs (TalncRNA73 and TalncRNA108) in three genotypes is closely related to stripe rust susceptibility [51]. Meanwhile, two porcine lncRNAs (linc-sscg2561 and Dnmt3a) showed differential expression levels between domesticated pigs and wild boars, suggesting a possible role for IncRNAs in pig domestication [52]. Maize domestication also reportedly reshaped the transcriptome, since DEGs are enriched in targets of selection during maize domestication and improvement [53]. Therefore, the differentially expressed lncRNAs we identified here could have important regulatory functions in meiosis, and, given the effect that the selection process has on recombination, may have been subjected to selection through sunflower domestication [34].

\section{Comparing the expression of IncRNAs in somatic and meiocyte transcriptomes}

To gain insight into the function of sunflower lncRNAs, we examined their expression behavior in the meiocyte and somatic transcriptomes of the domesticated sunflower genotype HA89 [17]. In HA89 meiocytes, the proportion of lncRNAs was significantly higher $(P<0.01)$ than that of the somatic transcriptome (Fig. 3a). Likewise, as mentioned above, high expression levels of lncRNAs in reproductive structures have been described in plants [46] and animals [43, 45]. Given the high number of lncRNAs expressed in sunflower meiocytes, many are likely involved in meiosis, and therefore the expression (and possible role) of lncRNAs in meiosis could be highly conserved. 
a

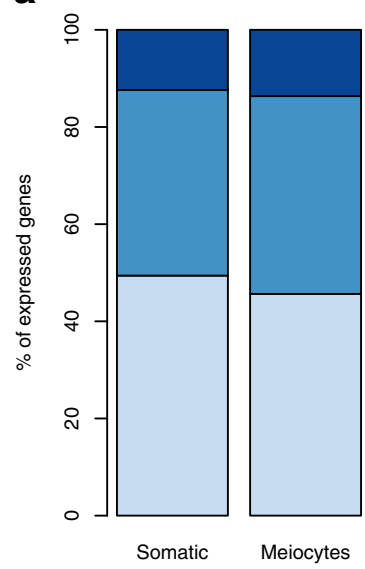

b

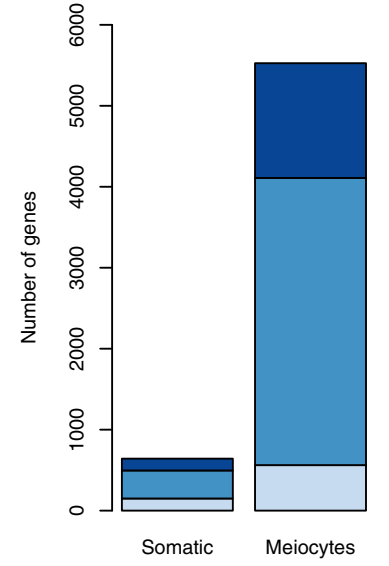

C

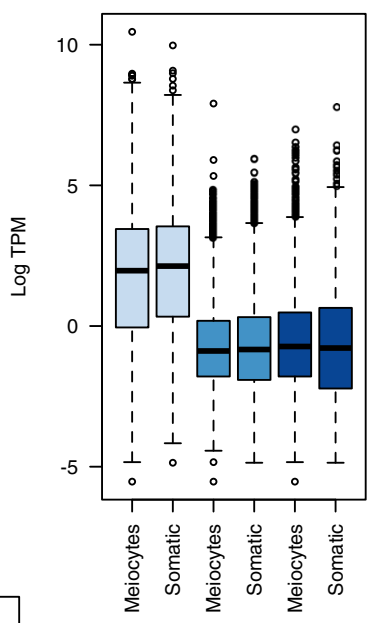

Fig. 3 Expression overview of genes grouped by coding class (Protein-coding, IncRNA or Unclassified) in somatic and meiocyte transcriptomes. a Bar chart showing the coding class proportions by transcriptome. b Bar chart comparing the number of genes exclusively expressed in somatic or meiocyte transcriptomes by coding class. c Box plots for the distributions of the decimal log expression of genes in meiocytes and somatic transcriptomes by coding class

Furthermore, we found that the majority of transcripts that showed expression in only one of the two transcriptomes (exclusively expressed genes), were lncRNAs in both somatic and meiocyte transcriptomes (Fig. 3b), although the number of lncRNAs was higher for the meiocyte transcriptome. On the other hand, the expression level (in transcripts per million; TPM) of these lncRNAs was lower than that observed for protein-coding genes (Fig. 3c), suggesting that sunflower lncRNAs are also very tissue-specific and have lower expression levels than protein-coding genes. These two common characteristics of lncRNAs are consistent with findings for other organisms $[21,29,46]$.

\section{Small RNA (sRNA) populations in sunflower meiocyte transcriptomes and their relationship with meiotic IncRNAs}

According to Li et al. [46], more than the $90 \%$ of putative maize lncRNAs have sequence similarity with small RNAs (sRNA), which they classified as pre-lncRNAs. Additionally, in other model species such as rice, Arabidopsis, and Populus trichocarpa, lncRNAs could be precursors [47, 54] or mimic targets of sRNAs [48, 55]. Thus, to characterize the connection between noncoding sRNAs and lncRNAs, we sequenced the sRNA transcriptome of prophase I meiocytes from wild and domesticated sunflower genotypes.

We obtained around 5 million (Table AF1-2 in Additional file 1) clean reads of 20 to $25 \mathrm{nt}$, with most corresponding to reads of $24 \mathrm{nt}$ (Fig. 4). These $24 \mathrm{nt}$
sRNAs are typically endogenous siRNAs [27] and are the major component of sRNA populations in plants that participate in RNA-mediated chromatin-based gene silencing [56]. During maize meiosis, 24 nt phasiRNAs accumulate [30] in a way that is similar to the accumulation observed for mouse spermatogenesis [31]. Although the function of these $24 \mathrm{nt}$ sRNAs is not completely understood, they may participate in genome surveillance (e.g., TE silencing pathways), or act as mobile signals and/or chromatin modifiers [30, 31].

We previously found that gene silencing pathway genes are highly expressed in meiocytes, especially the ortholog of the Arabidopsis AGO4 protein, which participates in $24 \mathrm{nt}$ siRNA binding. We found that meiocyte expression of the sunflower AGO4 ortholog was increased by 2.9-fold relative to the somatic transcriptome [17]. Given that Arabidopsis AGO4 is known to preferentially bind 24 nt siRNAs with a $5^{\prime}$ adenosine [57], we examined $5^{\prime}$ terminal nucleotide bias in sunflower $24 \mathrm{nt}$ sRNAs. A majority of sunflower 24 nt sRNAs indeed had a 5 ' terminal adenosine (Figure AF1-2 in Additional file 1), suggesting that these 24 nt sRNAs could be loading in the sunflower AGO4 orthologous complex, and thus could participate in maintaining silent states at repeated loci, transposons, and heterochromatin, in a similar manner to that of Arabidopsis [58]. The description of these abundant 24 nt sRNAs in sunflower meiocytes helps to support the idea of a convergent evolution of sRNA systems that regulate male reproductive development $[30,31]$. 
a. Wild

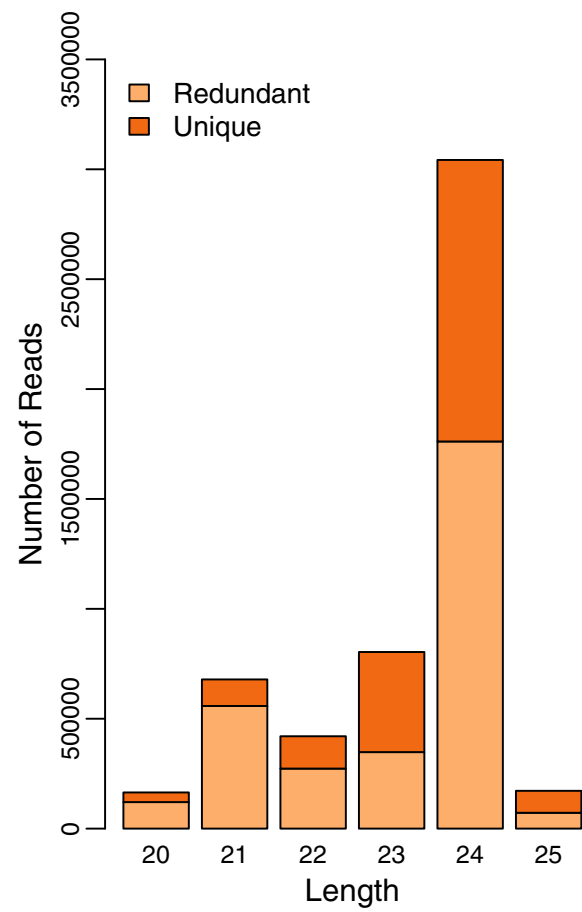

b. Domesticated

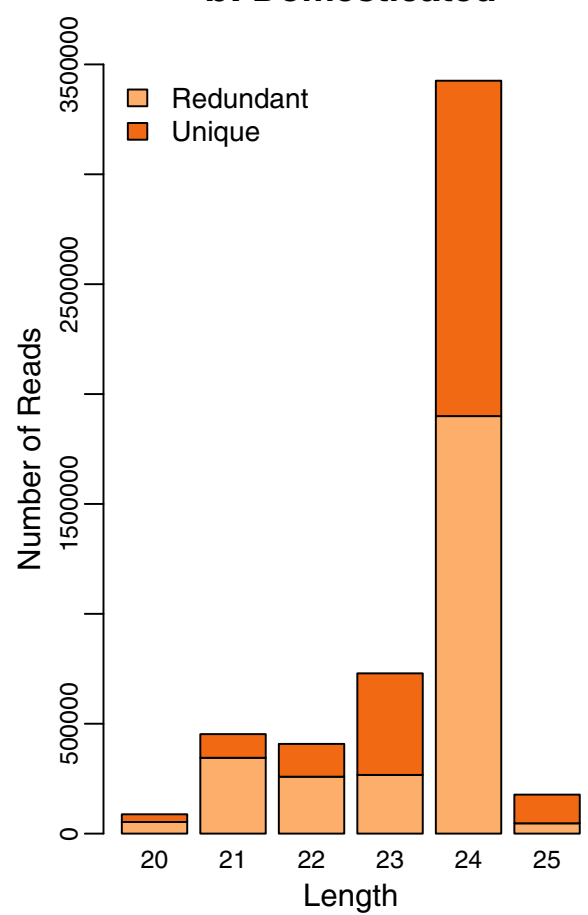

Fig. 4 Bar charts for the number of reads per length (bp) in small RNA populations of sunflower meiocytes in two genotypes. Reads were classified as 'Unique' for sequences found only once, or 'Redundant' for sequences found more than one time within genotypes. a Results for the wild genotype Ac-8. b Results for the domesticated genotype HA89

Consistent with observations in other plant species [59-61], the 24 nt sRNA population in sunflower is less redundant than the $21 \mathrm{nt}$ population (Fig. 4). sRNA with 24 nt have a higher number of unique or low abundance reads, while the 21 nt population includes multiple copies of the same sRNA. Moreover, the 21 nt sRNA population in plants is usually composed of miRNAs, which help regulate gene expression through post-transcription gene silencing mechanisms [62].

The expression of 32 different miRNA families was detected in both wild and domesticated genotype meiocytes (Figure AF1-3 in Additional file 1), but $92.69 \%$ and $85.87 \%$ of the reads for the domesticated and wild genotypes, respectively, were one of only three different 21 nt miRNA (miR166, miR396, and miR319) that show high conservation among terrestrial plant species [62]. Although the function of these three miRNAs in meiosis is unknown, in tomato miR396 and miR166 are differentially expressed between wild type and male-sterile mutant $7 B-1$ anthers, suggesting that they may have a role in anther development and male fertility [63]. Meanwhile, miR319 is expressed in the Arabidopsis male germline [64]. Here the relative abundance of these miRNA families was almost the same in the wild and domestic genotypes (Figure AF1-3 in Additional file 1), with the exception of miR398, which was 7-fold more abundant in the wild meiocytes. In cotton, miR398 was also differentially expressed during meiosis and tetrad stages of anthers from wild type and genetic male sterility (GMS) mutant plants [65]. The above finding indicates that the role of mi398 in meiosis merits additional study.

To establish the relationship between sRNA populations and lncRNAs, we mapped sRNAs against a mixed reference (contigs of the genome draft and the transcriptome assembled for this study), which allowed us to avoid selection bias, that is, to assign a transcript as a precursor or target of a sRNA, when the best hit for that sRNA is in a non-transcribed intergenic region (See Methods). Most 21 nt sRNAs mapped to protein coding transcripts, while the proportion of $24 \mathrm{nt}$ that mapped to protein-coding transcripts was similar to that which mapped to lncRNAs (Fig. 5). sRNA reads of the wild genotype mapped to 9370 lncRNAs, while the domesticated genotype sRNA reads mapped to 8852 lncRNAs (42.69\% and $40.91 \%$, relative to the total lncRNAs expressed in each genotype). sRNAs reads for both genotypes mapped to the same 8852 lncRNAs. Even though the proportion of lncRNAs having sequence similarity with miRNAs is notable, it is not as high as that for maize [46]. 

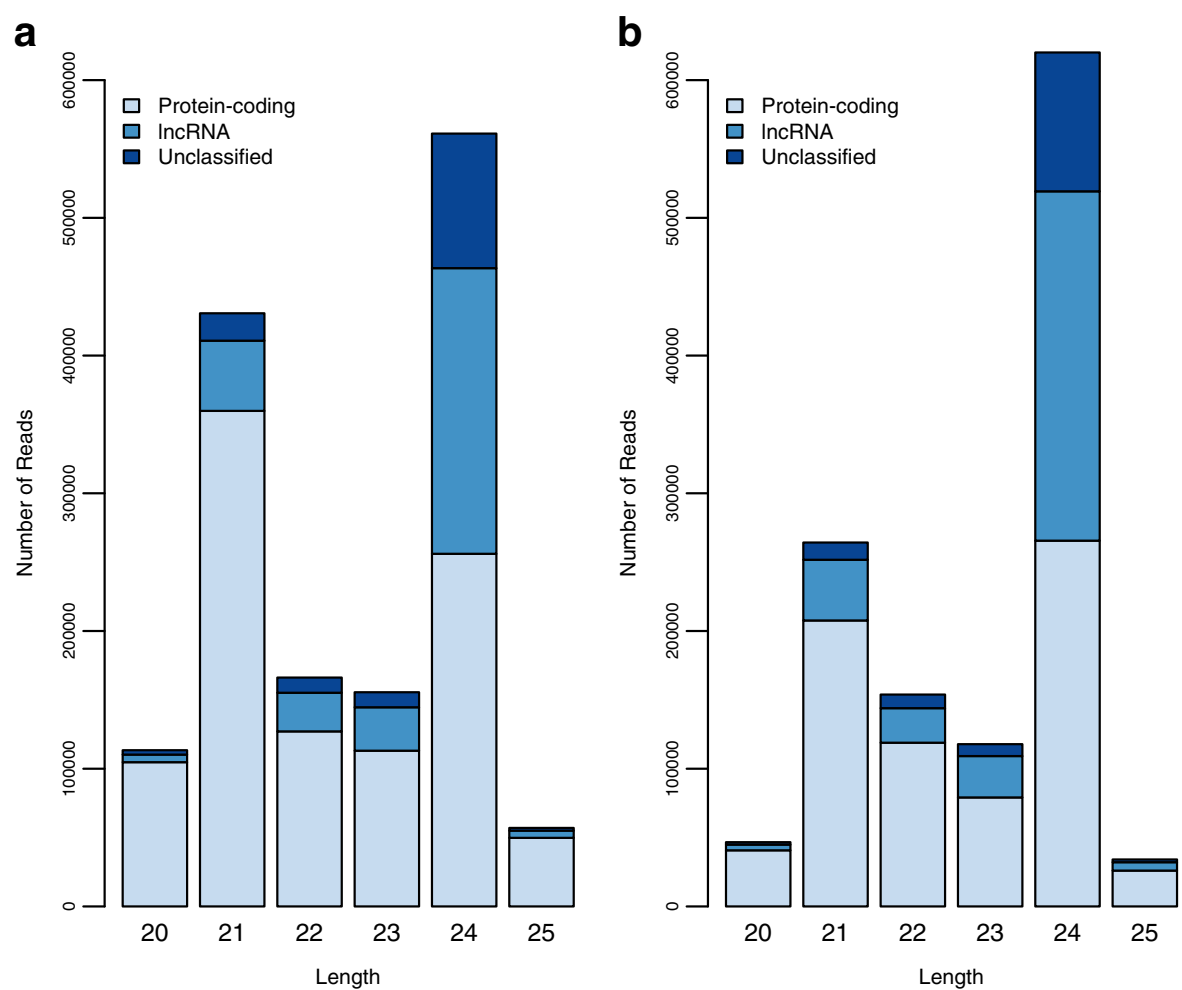

Fig. 5 Bar charts for the number of reads per length $(b p)$ in small RNA populations of sunflower meiocytes mapping to protein-coding, IncRNA or unclassified transcripts in two genotypes. a Results for the wild genotype Ac-8. b Results for the domesticated genotype HA89

According to Axtell [27], the patterns of referencealigned sRNAs provide information about sRNA biogenesis. We found that 20 and 21 nt sRNAs showed major differences ( $15 \%$ more reads mapped to the ' + ' strand) in the proportion of reads that mapped to the lncRNA '+' or '-' strand (Figure AF1-4 in Additional file 1). This result is expected, considering that these sRNA sizes are associated with miRNAs, which are generated through fragmentation of a single-stranded precursor, while $24 \mathrm{nt}$ (mostly siRNAs) arise from double-stranded RNA precursor cleavage. Thus, siRNAs are indeed the major sRNA population in sunflower meiocytes, and many lncRNAs are siRNA precursors.

On the other hand, according to BLAST searches against sunflower natural antisense transcripts (NATs) in the PlantNATs public database (See Methods), we determined that 388 IncRNAs are trans-natural antisense transcripts (trans-NATs). Of these trans-NATs, 198 and 215 for domesticated and wild type genotypes, respectively, could be cataloged as sRNA precursors, since sRNAs reads mapped to these NATs. Furthermore, $68.37 \%$ and $68.68 \%$ of NATs for wild and domesticated genotypes, respectively, are related to $24 \mathrm{nt}$ sRNAs. Other sRNA lengths were also related to NATs, albeit in lower proportions (Figure AF1-5 in Additional file 1).
Thus, some NAT-siRNAs are also active in sunflower meiocytes and could be part of regulatory mechanisms for gene expression that involve transcript cleavage, as was previously described for these types of sRNA $[66,67]$.

Together our results indicate that there is a complex regulatory network of sRNAs-lncRNAs working at the transcriptomic level in sunflower meiocytes. Considering that various sunflower genotypes show differences in lncRNA expression, lncRNAs are likely relevant to the regulation of recombination rates. However, some IncRNAs appear to be unrelated in sRNA regulatory pathways, so they may be involved in regulating transcription through other mechanisms.

\section{Repetitive elements in sunflower IncRNAs}

Transposable elements (TEs) are thought to be important contributors to the origin, evolution, and function of lncRNAs [68]. Through analysis of different regions of long intergenic non-coding RNAs (lincRNA) in mice and humans, Kannan et al. [69] found that the TE content of lincRNA genes is higher than in protein-coding genes, and that most TEs are present in the exons and promoter regions of lincRNAs. They also observed a correlation between TE insertion and the evolutionary rate of lincRNAs (e.g., there was more TE fixation in 
fast-evolving lincRNA genes). On the other hand, in tomato the insertion of a long terminal repeat (LTR) retrotransposon was important in the origin of the fruitspecific lncRNA $\ln R N A-314$, which presumably was generated during tomato domestication given its potential involvement in fruit ripening [70].

We found that $2326(9.18 \%)$ of all lncRNAs identified here contained TEs (Fig. 6). Of these, $91.01 \%$ also carried retrotransposons, while the remaining $8.99 \%$ had DNA transposons (Fig. 6a). The most common TE belonged to the long terminal repeat (LTR) retrotransposons from the Gypsy and Copia families (Fig. 6b). These results are consistent with previous studies showing that these two retrotransposon families are also the more abundant in the sunflower genome [71, 72]. Other repetitive elements, such as tandem repeats and unknown repeats, were also identified (Figure AF1-6 in Additional file 1). The percentage of lncRNAs that contained transposable or repetitive elements was $14.31 \%$, which was lower than that for maize [46]. Although the different lncRNA TE content in maize and sunflower could be due to methodological strategy, they may also be related to differences in genomic TE contents of the tissues from which the IncRNAs were sampled (i.e., meiotic expressed lncRNAs in sunflower vs. whole-plant expressed lncRNAs in maize), or intrinsic characteristics of IncRNAs. Even in maize, the majority of so-called
HC-lnRNAs (those that are not sRNA precursors) do not contain repetitive sequences [46], which suggests that lncRNA diversity occurs at both functional and evolutionary levels, even within the same species. As such, inter-species differences in IncRNA characteristics would certainly be expected.

The presence of TEs in sunflower lncRNAs is an interesting result, since these elements could contribute to an understanding of their function and biology. For instance, TE-related variability was reduced among domesticated plants compared to wild $H$. annuus accessions [72]. Moreover, the involvement of TE in domestication and trait improvement has also been reported for several plants, including maize and wheat [73], indicating that the TE content between wild and domesticated plants is dynamic. Thus, the domestication process can create a permissive environment for the generation of new or altered lncRNAs that in turn affect regulatory networks by fostering phenotypic changes (e.g., differences in the recombination rate) in a relatively short timeframe.

One surprising finding of transcriptome studies in Arabidopsis meiocytes [14, 15] was the high expression of TEs, which could generate mutations or have harmful effects on the genome or germline that could be inherited by future generations [13]. Thus, maintenance of the structural integrity of a plant's genetic material is critical. Although transposon expression may be an

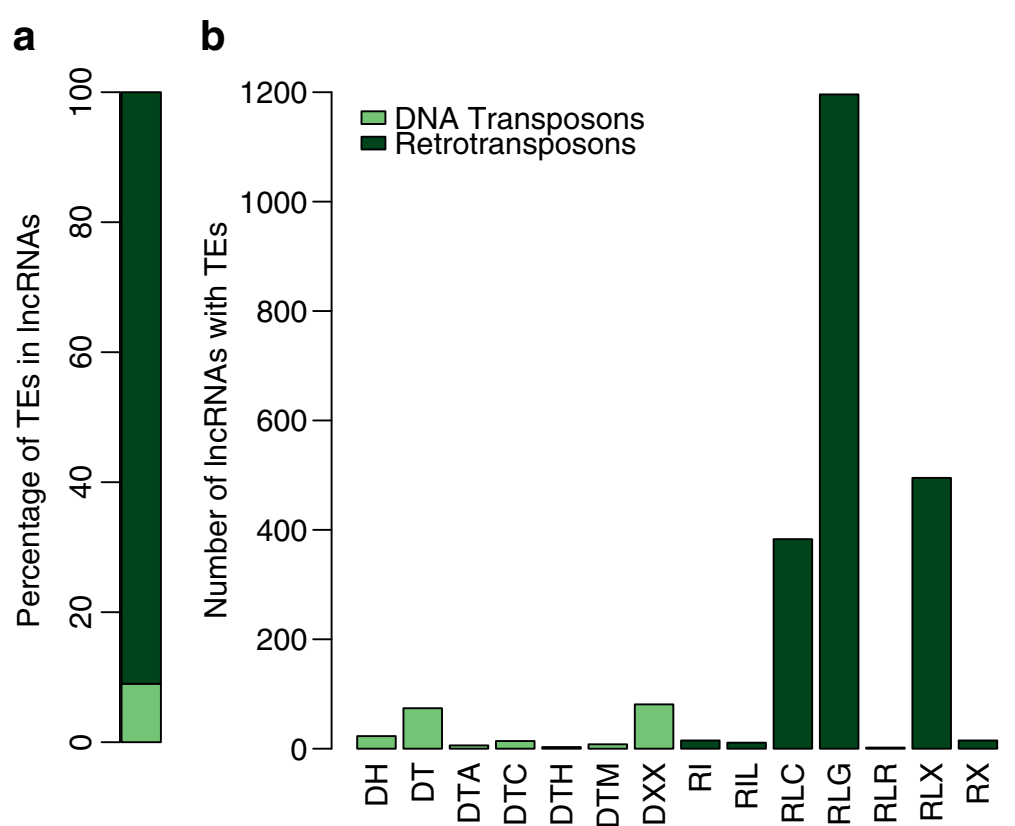

Fig. 6 Transposable elements (TES) in sunflower IncRNAs. a Relative percentages of DNA Transposons and Retrotransposons found within 2326 (9.18 \%) sunflower IncRNA. b Bar chart for the number of IncRNA containing TEs per TEs family. DNA Transposons: DH = Helitron, DT = Unknown DNA transposon, DTA $=$ hAT, DTC $=C A C T A, D T H=$ Harbinger, DTM $=$ Mutator, DXX $=$ MITE. Retrotransposons: RI = unknown non-LTR retrotransposons, RIL = LINEs, RLC = Copia retrotransposons, RLG = Gypsy retrotransposons, RLR = retroviruses, RLX = Unknown LTR retrotransposons, $\mathrm{RX}=$ Unknown retrotransposons 
unintended result of chromatin structure reorganization during meiosis [13], their expression could also have a functional role, especially since TEs can participate in chromatin remodeling by recruiting silencing machinery [74]. Thus, although some TE expression in meiocytes is likely genuine, which would be justified by the high expression of proteins such as AGO9 that participate in TE silencing mechanisms during formation of female gametes [75] and the siRNA silencing pathways described above [30,31], some meiocyte TEs could actually be functional domains of IncRNAs [68]. This possibility implies that TEs perform their role in chromatin remodeling during meiosis, and their integration in lncRNAs would not compromise genomic integrity. Even though future analyses are needed to test this hypothesis, the presence of TEs in lncRNAs that are highly expressed in meiocytes opens a new perspective that could yield clues about their highly counterintuitive expression in the germ line.

\section{Identification of putative targets for IncRNAs}

The identification of molecules that interact with the lncRNAs, could be also important to elucidate their function. One of the possible functions of IncRNAs is regulation of protein-coding transcripts through RNA/ RNA interactions [24]. With the aim of predicting putative protein-coding targets for the set of lncRNAs found in this work, we tested the 'LncTar' algorithm [76], on a subset of lncRNAs and sunflower meiotic genes. In this experiment we found some promising results, as the fact that three lncRNAs have a predicted significant interaction with sunflower meiotic gene MMD1, involved in spindle cytokinesis, and also these three lncRNA present a highly correlated change of relative expression with MMD1 in the three genotypes $(r>0.98$; see Tables AF16 and AF1-7 and Figure AF1-1 in section "Putative Targets for lncRNAs" in Additional file 1). Given that in silico prediction of RNA/RNA interaction, as detected by LncTar, is only one of the factors to take into account to predict lncRNA/target interactions, the above example and many more detected (see "Putative targets for lncRNAs" in Additional file 1), need further experimental confirmation to be taken into account in the understanding of individual lncRNAs functions.

\section{Final remarks on sunflower meiotic IncRNAs}

The idea that lncRNAs act as key gene regulators is supported by several features such as: i) immediate functionality upon transcription (i.e., no need for translation into protein); ii) versatile structural or sequence-specific interactions with proteins or nucleic acids; and iii) evolutionary flexibility that promotes tolerance of insertions or deletions given that their function is independent of a strict sequence frame like that of protein-coding genes
[68]. These features are consistent with increasing evidence suggesting that IncRNAs are involved in many different biological processes, and thus expand our knowledge of the regulation of certain processes and/or alter some paradigms [21, 25]. A meeting point of studies describing genome-wide identification of lncRNAs is that IncRNAs are highly expressed in sexual reproduction structures in both animals [45] and plants [46]. Although the relationship of lncRNAs to plant fertility is also established [29, 41, 42], as is the induction of $21 \mathrm{nt}$ phasiRNAs in rice meiosis [47], no plant meiosis-specific lncRNA has yet been described [13]. So, to the best of our knowledge, this is the first report of meiosis-specific lncRNAs in plants, which open questions about their function and importance in regulating fundamental sexual reproduction processes.

To explore the possible role of these meiosis-specific lncRNAs in sunflower meiocytes, we compared them with lncRNAs expressed in the somatic transcriptome (Table 1). The meiosis-exclusive lncRNAs had significantly more hits in the sunflower genome $(87.18 \%$ vs. $64.77 \%, P<0.01)$, greater differential expression (58.22\% vs. $50.49 \%$ ), a larger degree of sRNA similarity (36.77\% vs. $23.33 \%$ ), and a higher TE content (8.22 \% vs. $6.46 \%)$ than IncRNAs expressed in both meiocyte and somatic transcriptomes. These meiocytespecific differences give valuable clues about lncRNA function in meiosis. First, the larger number of genome hits suggests greater conservation, which is in agreement with previous observations in tetrapods, where lncRNAs related to spermatogenesis displayed a higher level of conservation [45]. Second, some meiosis-specific lncRNAs could play a larger role in the recombination rate relative to non-specific lncRNAs. Finally, their higher similarity with sRNAs and higher TE content suggest that many lncRNAs may promote chromatin state modifications, especially given the differences in chromatin reorganization during meiosis $[43,77,78]$ and that sRNAs and TEs are thought to have regulatory roles in chromatin modification [74, 79].

We also observed differences in lncRNA expression between domesticated and wild genotypes. In lncRNAs with exclusive expression, the proportion of nondifferentially expressed lncRNAs with genome hits was lower than that for differentially expressed lncRNAs (43.34 \% vs. $56.66 \%$ ), while for non-exclusive lncRNAs the opposite tendency was observed (higher proportion in non-differentially expressed lncRNAs, $52.94 \%$ vs. $47.06 \%)$. These differences may provide evidence that meiocyte-specific lncRNAs could be more closely related to differences in the recombination rate than nonmeiocyte specific lncRNAs, and that the major proportion of these lncRNAs are more conserved or do not have splicing modifications (which explains the higher 
Table 1 Summary of sunflower IncRNA features. The total number of IncRNAs having each feature is presented. Percentages of differentially and non-differentially expressed IncRNAs were calculated according to the total of IncRNAs with a given feature (values in the total column). Percentages in the total for each feature (total column) were calculated with respect to the total number of IncRNAs independently of features (value in the total row and total column)

\begin{tabular}{|c|c|c|c|}
\hline & Not differentially expressed & Differentially expressed & Total \\
\hline \multicolumn{4}{|l|}{ Somatic and meiocytes expressed } \\
\hline Genome hit & 8925 (49.72 \%) & $9024(50.28 \%)$ & $17,949(70.87 \%)$ \\
\hline Expression in three genotypes & $8250(52.65 \%)$ & $7421(47.35 \%)$ & $15,671(61.87 \%)$ \\
\hline Expression in three genotypes and genome hit & $8083(52.80 \%)$ & $7227(47.40 \%)$ & $15,310(60.45 \%)$ \\
\hline sRNAs similarity & 3035 (44.40 \%) & $3800(55.60 \%)$ & $6835(26.99 \%)$ \\
\hline Contains transposons & $831(47.25 \%)$ & $928(52.75 \%)$ & $1759(6.94 \%)$ \\
\hline Total of IncRNAs identified & $12,006(47.40 \%)$ & $13,321(52.60 \%)$ & 25,327 \\
\hline \multicolumn{4}{|l|}{ Meiocytes 'exclusive' } \\
\hline Genome hit & $2605(43.34 \%)$ & 3406 (56.66 \%) & $6011(87.18 \%)$ \\
\hline Expression in three genotypes & $1564(53.97 \%)$ & $1334(46.03 \%)$ & $2898(42.03 \%)$ \\
\hline Expression in three genotypes and genome hit & $1520(53.94 \%)$ & $1298(46.06 \%)$ & $2818(40.87 \%)$ \\
\hline sRNAs similarity & $1081(42.64 \%)$ & $1454(57.36 \%)$ & 2535 (36.77 \%) \\
\hline Contains transposons & 279 (49.21\%) & $288(50.79 \%)$ & $567(8.22 \%)$ \\
\hline Total of meiocytes 'exclusive' expressed & $2881(41.78 \%)$ & $4014(58.22 \%)$ & 6895 \\
\hline \multicolumn{4}{|l|}{ Somatic and meiocytes expressed (meiocytes 'exclusive' not included) } \\
\hline Genome hit & $6320(52.94 \%)$ & $5618(47.06 \%)$ & $11,938(64.77 \%)$ \\
\hline Expression in three genotypes & $6686(52.34 \%)$ & $6087(47.66 \%)$ & $12,773(69.33 \%)$ \\
\hline Expression in three genotypes and genome hit & $6563(52.54 \%)$ & $5929(47.46 \%)$ & $12,492(67.77 \%)$ \\
\hline sRNAs similarity & $1954(45.44 \%)$ & $2346(54.56 \%)$ & $4300(23.33 \%)$ \\
\hline Contains transposons & $552(46.31 \%)$ & $640(53.69 \%)$ & $1192(6.46 \%)$ \\
\hline $\begin{array}{l}\text { Total of IncRNAs expressed in somatic and meiocyes, excluding } \\
\text { meiocytes 'exclusive' expressed }\end{array}$ & $9125(49.51 \%)$ & $9307(50.49 \%)$ & 18,432 \\
\hline
\end{tabular}

number of genome hits). We also found that lncRNAs with differential expression have higher sRNA similarity in both meiocyte-specific and non-meiocyte-specific lncRNAs, although this difference is more notable for the specific lncRNAs $(57.36 \%$ vs. $42.64 \%$ and $54.56 \%$ vs. $45.44 \%$ for meiocyte-specific and non-meiocyte specific lncRNAs, respectively). Since lncRNAs with more variable expression were mostly related to sRNAs, the linked chromatin modification function of lncRNAssRNAs may provide the most significant contribution to the differences in recombination rates. Lastly, differences in the proportion of differentially and non-differentially expressed lncRNAs that contain TEs were observed. In meiocyte-specific lncRNAs the proportion of nondifferentially expressed lncRNAs with TEs was slightly lower than that for differentially expressed lncRNAs (49.21\% vs. $50.79 \%)$. However, for non-meiocytespecific lncRNAs we observed that this difference is wider (46.31\% vs. $53.69 \%$, non-differentially and differentially expressed lncRNA, respectively).. Further studies will be needed to clarify the role of these TE-containing lncRNAs in meiosis, and also to establish if their function is related to the higher TE frequency in meiocytes $[14,15]$.

\section{Conclusions}

In recent years growing evidence suggested that lncRNAs have a wide range of important regulatory functions [21-23, 38]. In particular, genome-wide identification and characterization of lncRNAs in different plant and animal species spotlighted their high expression in reproductive structures such as testicles and anthers $[29,43,45,46]$, and that these IncRNAs have a greater degree of conservation that is suggestive of their key role in sexual reproduction [45]. Although the involvement of lncRNAs in sexual reproduction and fertility in plants $[29,41,42]$ is well documented, whether lcRNAs also function in plant meiosis was unclear [13], and no plant meiosis-specific lncRNAs were known. Here, we obtained transcriptomes of meiocytes in prophase I from three different sunflower genotypes that showed different recombination rates. Through sequencing we could obtain a complete transcriptome with no missing genes [35], with which we generated results 
indicating that a major proportion of DEGs were lncRNAs, some of which were expressed exclusively in meiocytes.

Our data suggest that, relative to non-meiosis specific lncRNAs, meiosis-specific lncRNAs are more conserved within related genotypes (a major proportion had a genome hit), have greater expression variability in meiocytes from wild and domesticated genotypes, and have a closer relationship with elements such as siRNAs and TEs that are related to chromatin remodeling. These results highlight the fundamental role of IncRNAs in meiosis, and suggest a connection between two features of meiocytes and/or anthers: high TE activity and higher frequency of siRNAs. We also found evidence to support a role for lncRNAs in meiotic functions [13], such as maintenance of heterochromatin and influencing epigenetic factors.

Although whether lncRNAs directly or indirectly affect meiotic homologous recombination, or whether other factors could explain the differences in the recombination rate is unclear, the meiosis-specific differentially expressed lncRNAs identified here may be involved in processes that led to these phenotypic differences. For instance, some lncRNAs are associated with the advent of domestication features [52] or were modified during domestication [70], suggesting that the strong artificial selection that occurs during domestication could influence lncRNAs. Likewise, lncRNAs are both widely conserved and rapidly evolving elements [45, 46, 80], and therefore may represent a rich source for evolutionary innovations [79] that allow greater flexibility in selection processes without compromising sexual reproduction, or for regulatory changes in meiosis that do not affect essential genetic elements.

Future efforts will focus on determining whether sunflower-specific or preferentially expressed lncRNAs are conserved in other plant species, and in characterizing their activity at a functional level. These studies will require the generation and re-analysis of genomic data and meiocyte expression patterns and/or reproductive structures in other plants, as well as the completion of the sunflower genome and advances in functional genomic tools in this plant.

\section{Methods}

\section{Meiocyte collection, RNA extraction, and sequencing}

Sunflower plants of three genotypes: domesticated (inbred line HA89, Helianthus annus L. var. macrocarpus), F1 (F1 generation cross between domesticated and wild genotypes) and wild (Ac-8, Helianthus annuus L. ssp. texanus), were grown under greenhouse conditions as previously described [17]. At the beginning of the R2 development stage [81], approximately 10 disc florets of the floral bud were squashed with dissecting needles on a concave glass slide with $80 \mu \mathrm{L}$ sterile distilled water. A first filter to confirm the meiotic stage was performed under a microscope (without staining) to determine whether the meiocytes remained associated to form the characteristic "worm" structure of prophase I meiocytes [15]. If the meiocytes appeared to be in early meiotic stages and pollen grains or tetrads were absent, a subsample of the disc floret was fixed in a $96 \%$ ethanol:acetic acid solution (3:1) for $24 \mathrm{~h}$, and then observed under a microscope with the squashedacetocarmine staining method to confirm the meiotic phase. Once samples passed this 'double-check' proto$\mathrm{col}$, meiocytes were collected from developmentallymatched florets in RNAlater (Ambion, Inc.) and stored at $-70^{\circ} \mathrm{C}$ until RNA extraction.

RNA of prophase I meiocytes from each genotype was isolated using the ZR RNA MicroPrep kit (Zymo Research, Orange, CA) following the manufacturer's instructions, and stored at $-70{ }^{\circ} \mathrm{C}$. Six libraries from meiocytes (two biological replicates for each genotype) were prepared using standard Illumina TruSeq RNA library preparation kits, and sequenced using the Illumina HiSeq 2500 platform to obtain 100 bp paired-end reads.

\section{Quality filtering, de novo assembly, and gene expression estimation}

Adaptors were removed from the reads using cutadapt 1.3 software [82]. Adaptor-free reads were subsequently quality-trimmed using PRINSEQ 0.20.4 software [83], allowing a minimum quality score of 20 and no more than two ambiguous bases per read. Then, de novo assembly of the trimmed reads was performed using Trinity (release 20140413) software [84] and default parameters. The assembler classifies the output in two categories: 'genes', corresponding to sequences that the algorithm considers are the product of different genes, and 'transcripts', which contain sequences with slight differences between them so as to be cataloged as a different gene; these could be splice variants or distinct alleles. Thus, to quantify the expression, we selected the longest sequence of each reconstructed 'gene' and remapped the reads using Bowtie2 2.1.0 (set-up parameters: -a -rdg 6, 5 -rfg 6, 5 -score-min L, -0.6, -0.4) [85]. Those reads that mapped exclusively to one gene (unique read counts) were estimated using eXpress 1.4.1 [86] with default parameters. These counts were arranged in a matrix for subsequent analysis.

\section{Gene identification}

Transcripts from protein coding genes present in the assembled transcriptome were identified by sequentially querying them to four different peptide databases using blastx [87], which translates the six reading frames of the transcript to the corresponding peptide sequences, and then look for significant similarities with the peptide 
database. Hits were considered significant if the bitscore of the alignment was $\geq 90$ and had an expected value $\mathrm{E} \leq 10^{-6}$. The use of a threshold of bit-score $\geq 90$ guarantees a minimum average alignment of approximately 100 aa $(300 \mathrm{bp})$, and thus is very likely to correctly identify the peptide coded by a transcript. First, transcripts were compared against $A$. thaliana (TAIR10) peptides, and transcripts that passed the above threshold where considered as protein-coding and identified by the corresponding A. thaliana ortholog. Transcripts without a significant hit where compared, in turn, with sunflower peptide dataset for varieties HA412 and HAXRQ, available at the HeliaOrg website (https:// www.heliagene.org) and finally with NCBI RefSeq plant peptides (release 24/07/2013). At each step sequences having a significant hit (bit-score $\geq 90$ and $\mathrm{E} \leq 10^{-6}$ ) were considered as protein coding and identified with the corresponding peptide, and only the ones without a significant hit were used in the next steps. We used first TAIR10 given that $A$. thaliana has the best curated set of plant genes and proteins. Transcripts without a significant hit to TAIR10 were compared to sunflower peptide databases to account for peptides specific to this genus and with no ortholog in A. thaliana, while comparison with RefSeq plant peptides covered the possibility of peptides missed from the previous databases. Genes having no hits among any of the queried peptide databases were cataloged as 'unidentified' and used for subsequent lncRNA identification analyses. A MySQL relational database (Server version 5.5.34) that included all data from assembly, mapping, and annotation was also compiled.

\section{Identification of IncRNA}

Since all unidentified transcripts had a length $>200 \mathrm{nt}$, length filtering was not necessary. Two different algorithms were used to determine if a transcript could be the product of a lncRNA: a coding protein calculator analysis tool in the CPC web interface (http:// cpc.cbi.pku.edu.cn) [36] and analysis with the CodingPotential Assessment Tool (CPAT) [37]; default parameters were used for both. For the CPAT analysis, the protein coding gene models were constructed using the A. thaliana genome (TAIR10). Only genes that passed the threshold for both analyses (CPC score $\leq-1$ and CPAT score $\leq 0.3$ ) were cataloged as lncRNAs. Even when the nominal threshold for CPAT score was set to $\leq 0.3$, all the IncRNAs reported here have a very low value of protein coding probability, CPAT score $\leq 1 \times 10^{-6}$ (Table AF1-4 in Additional file 1), and the simultaneous application of the two algorithms excluded 7479 (21.80\%) sequences as putative IncRNA (Table AF1-3 in Additional file 1). We also show that our criteria to catalogue genes as lncRNAs are at least as stringent as the ones used in 17 recent references mentioning lncRNA detection (see Table AF1-5 and section "Additional discussion of IncRNA Identification" in Additional file 1). We also did a blastn analysis of the identified lncRNAs with the genome draft assembly of the sunflower inbred line HA412 (Celera_14libs_sspace2_ext. final.scaffolds.split.fasta) deposited in the Sunflower Genome Project website: http://www.sunflowergenome.org, to which Prof. Loren Rieseberg (University of British Columbia) kindly gave us access. A hit with the genome was considered significant if the result had a bitscore $\geq 90$ and an expected value $\mathrm{E} \leq 10^{-6}$.

\section{Identification of putative targets for IncRNAs}

Software package 'LncTar' [76] (version of September-01, 2015) was downloaded from site http://www.cuilab.cn/ lnctar, and run in a subset of the sunflower lncRNAs and meiotic genes. Details of the procedure and results are presented in section "Putative targets for lncRNAs" of Additional file 1.

\section{Small RNA sequencing and mapping}

RNA from prophase I meiocytes from domesticated and wild genotypes was isolated using the ZR RNA MicroPrep kit (Zymo Research, Orange, CA) following the manufacturer's instructions and modifications for sRNAs extractions, and stored at $-70{ }^{\circ} \mathrm{C}$. Two libraries (one for each genotype) were prepared using standard Illumina TruSeq Small RNA library preparation kits, and sequenced using the Illumina HiSeq 2500 platform to obtain $37 \mathrm{bp}$ single-end reads. Reads were quality-trimmed using the Kraken set of tools [88], and those reads with a length between 20 and $25 \mathrm{nt}$ were selected for subsequent analyses. Mapping of the sRNA reads was performed using the Bowtie 1.1.2 version [89] to a mixed reference of the genome draft contigs (described above) and meiocyte transcriptome assembly from this study with the following parameters: -v 1 -best -strata -a -f -chunkmbs 512.

\section{Description of IncRNA characteristics: NATs and Transposons (TEs)}

To identify lncRNAs that were natural antisense transcripts (NATs), we downloaded the sequences of the predicted Helianthus annus natural antisense transcripts from the PlantNATsDB (http://bis.zju.edu.cn/pnatdb/) [90], and queried our lncRNAs against them using blastn. To identify TEs and repetitive sequences in the IncRNAs, we did a blastn search against sequences in the sunflower repetitive sequences database SUNREP [71], RepBase (Version 21.02) [91] and PGSB Repeat Element Database (PGSB-REdat) [92]. BLAST cutoff values were the same for both analyses: bit-score $\geq 70$ and expected value $\mathrm{E} \leq 10^{-6}$. Additionally, we conducted a search of repetitive elements 
in our lncRNAs by using the Web Server tool of RepeatMasker Version open-4.0.5 (http://www.repeatmasker. org/cgi-bin/WEBRepeatMasker) (A.F.A. Smit, R. Hubley and P. Green, unpublished), with the 'slow' option, in order to have the maximum sensitivity.

\section{Statistical design and analyses}

To perform differential expression analyses, we did two biological replicates for each sunflower genotype with two different sets of plants (floral buds, florets), and performed independent RNA isolation, library construction, and sequencing. Expression variations found for each gene between the two biological replicates gave an estimate of the statistical error (unexplained variation), which includes biological as well as technical variation. As previously described, the gene expression level was considered as the unique read counts obtained for each gene. However, for genes sharing the same peptide identifier, expression data were collapsed to a single gene by summing the numbers of reads that mapped to each component. We collapsed the counts according to their identifier in the following order: TAIR10, HA412, HAXRQ, and Refseq. To measure the number of missing genes in our transcriptome and to gauge completeness, we used the method described by García-Ortega and Martínez [35]. Differential expression analysis was made with the edgeR package [93], and the resulting p-values were input into the q-value function [94] with default parameters, setting the fdr.level $=0.01$ to obtain a FDR of $1 \%$. Differences in proportions were assessed with a two-tailed test for population proportion and a threshold of $P<0.01$. All statistical analyses were conducted in $\mathrm{R}$ version 2.15.3 [95].

\section{Additional file}

Additional file 1: Additional text, tables and figures. Text, Tables and Figures from this file are referred as "AF1-\#", where "\#" is the corresponding number in both, the main text and Additional file 1. (PDF 1223 kb)

\section{Acknowledgements}

We are grateful to Fernando Hernandez-Godínez, who modified and tested the meiocyte isolation protocol, helped cultivate plants, assisted in meiocytes collection and qRT-PCR analysis, and to Dr. Cei Leander Gastón Abreu-Goodger for advice and criticism for sRNA analyses. We also thank Prof. Loren Rieseberg from the University of British Columbia, and all members of the Sunflower Genome Project team, who gave us access to the draft sunflower genome sequence and to two anonymous reviewers by useful suggestions and constructive criticism.

\section{Funding}

This research was funded by grants from CONACYT (Consejo Nacional de Ciencia y Tecnología, Gobierno Mexicano) to Octavio Martínez (project 165778) and Nathalia M. V. Flórez-Zapata (scholarship 262855).

\section{Availability of data and materials}

All sequencing data are deposited in NCBI 'Sequence Read Archive' under submission number SUB1397228.
The public sunflower line HA89 was kindly provided by Dr. Gerald Seiler (USDA-ARS Northern Crop Science Laboratory). The seeds of $\mathrm{H}$. annuus ssp. texanus were obtained from an accession originated in the county of Saltiilo, state of Coahuila, Mexico. Seed recollection was performed in accordance with current Mexican regulations and based on ten heads from each of five sunflower plants. Seeds are deposited in the Laboratory of Genome Analysis of Universidad Autónoma Agraria Antonio Narro, with key 280-M2-2004. Field and greenhouse experiments were conducted in accordance with local legislation.

\section{Authors' contributions}

NMVFZ made substantial contributions to the research design, performed the bioinformatics analyses, interpreted the results, and wrote the first draft of the manuscript. MHRV collaborated on research design, suggested and validated the protocol for male meiocyte isolation, provided plant materials, and contributed to experimental design, as well as to the interpretation of the results, making important intellectual contributions and critically reviewing the manuscript. OM conceived and designed the research, directed the bioinformatics and statistical analyses, contributed to the interpretation of the results and wrote the final version of the manuscript. All authors have read the final version of the manuscript and agree with its content.

\section{Competing interests}

The authors declare that they have no competing interests.

\section{Ethics approval and consent to participate}

Not applicable.

\section{Author details}

${ }^{1}$ Laboratorio Nacional de Genómica para la Biodiversidad (LANGEBIO)/Unidad de Genómica Avanzada, Centro de Investigación y de Estudios Avanzados del Instituto Politécnico Nacional (Cinvestav), 36821 Irapuato, Guanajuato, México. ${ }^{2}$ Department of Plant Breeding, Universidad Autónoma Agraria Antonio Narro, Buenavista, 25315 Saltillo, Coahuila, México.

Received: 10 February 2016 Accepted: 25 May 2016

Published online: 11 July 2016

\section{References}

1. Armstrong SJ, Franklin FCH, Jones GH. A meiotic time-course for Arabidopsis thaliana. Sex Plant Reprod. 2003;16:141-9.

2. Hamant O, Ma H, Cande WZ. Genetics of meiotic prophase I in plants. Annu Rev Plant Biol. 2006;57:267-302.

3. Osman K, Higgins JD, Sanchez-Moran E, Armstrong SJ, Franklin FCH Pathways to meiotic recombination in Arabidopsis thaliana. New Phytol. 2011;190:523-44.

4. Carpenter A. Chiasma Function. Cell. 1994;77:959-62.

5. Vershon AK, Pierce M. Transcriptional regulation of meiosis in yeast. Curr Opin Cell Biol. 2000;12:334-9.

6. Mata J, Lyne R, Burns G, Bähler J. The transcriptional program of meiosis and sporulation in fission yeast. Nat Genet. 2002;32:143-7.

7. Primig M, Williams RM, Winzeler EA, Tevzadze GG, Conway AR, Hwang SY, Davis RW, Esposito RE. The core meiotic transcriptome in budding yeasts. Nat Genet. 2000;26:415-23.

8. Yang X, Makaroff CA, Ma H. The Arabidopsis MALE MEIOCYTE DEATH1 gene encodes a PHD-finger protein that is required for male meiosis. Plant Cell Online. 2003;15(June):1281-95.

9. Andreuzza S, Nishal B, Singh A, Siddiqi I. The Chromatin Protein DUET/ MMD1 Controls Expression of the Meiotic Gene TDM1 during Male Meiosis in Arabidopsis. PLoS Genet. 2015;11, e1005396.

10. Morohashi K, Minami M, Takase H, Hotta Y, Hiratsuka K. Isolation and characterization of a novel GRAS gene that regulates meiosis-associated gene expression. J Biol Chem. 2003;278:20865-73.

11. Pawlowski WP, Wang C-JR, Golubovskaya IN, Szymaniak JM, Shi L, Hamant O, Zhu T, Harper L, Sheridan WF, Cande WZ. Maize AMEIOTIC1 is essential for multiple early meiotic processes and likely required for the initiation of meiosis. Proc Natl Acad Sci U S A. 2009;106:3603-8.

12. Nan G-L, Ronceret A, Wang RC, Fernandes JF, Cande WZ, Walbot V. Global transcriptome analysis of two ameiotic1 alleles in maize anthers: defining steps in meiotic entry and progression through prophase I. BMC Plant Biol. 2011;11:120. 
13. Zhou A, Pawlowski WP. Regulation of meiotic gene expression in plants. Front Plant Sci. 2014;5(August):413.

14. Chen C, Farmer AD, Langley RJ, Mudge J, Crow JA, May GD, Huntley J, Smith AG, Retzel EF. Meiosis-specific gene discovery in plants: RNA-Seq applied to isolated Arabidopsis male meiocytes. BMC Plant Biol. 2010;10:280.

15. Yang $\mathrm{H}$, Lu P, Wang $\mathrm{Y}, \mathrm{Ma}$ H. The transcriptome landscape of Arabidopsis male meiocytes from high-throughput sequencing: the complexity and evolution of the meiotic process. Plant J. 2011;65:503-16.

16. Dukowic-Schulze S, Sundararajan A, Mudge J, Ramaraj T, Farmer AD, Wang M, Sun Q, Pillardy J, Kianian S, Retzel EF, Pawlowski WP, Chen C. The transcriptome landscape of early maize meiosis. BMC Plant Biol. 2014;14:118.

17. Flórez-Zapata NMV, Reyes-Valdés MH, Hernandez-Godínez F, Martínez O. Transcriptomic landscape of prophase I sunflower male meiocytes. Front Plant Sci. 2014;5(June):277.

18. Li J, Farmer AD, Lindquist IE, Dukowic-Schulze S, Mudge J, Li T, Retzel EF, Chen C. Characterization of a set of novel meiotically-active promoters in Arabidopsis. BMC Plant Biol. 2012;12:104.

19. Dukowic-Schulze S, Harris A, Li J, Sundararajan A, Mudge J, Retzel EF, Pawlowski WP, Chen C. Comparative Transcriptomics of Early Meiosis in Arabidopsis and Maize. J Genet Genomics. 2013.

20. Li J, Dukowic-Schulze S, Lindquist IE, Farmer AD, Kelly B, Li T, Smith AG, Retzel EF, Mudge J, Chen C. The plant-specific protein FEHLSTART controls male meiotic entry, initializing meiotic synchronization in Arabidopsis. Plant J. 2015;84:659-71.

21. Liu X, Hao L, Li D, Zhu L, Hu S. Long Non-coding RNAs and Their Biological Roles in Plants. Genomics Proteomics Bioinformatics. 2015;13:137-47.

22. Ariel F, Romero-Barrios N, Jégu T, Benhamed M, Crespi M. Battles and hijacks: noncoding transcription in plants. Trends Plant Sci. 2015;20:362-71.

23. Bonasio R, Shiekhattar R. Regulation of transcription by long noncoding RNAs. Annu Rev Genet. 2014:48:433-55.

24. Rinn JL, Chang HY. Genome Regulation by Long Noncoding RNAs. Annu Rev Biochem. 2012;81:145-66.

25. Cech TR, Steitz JA. The Noncoding RNA Revolution-Trashing Old Rules to Forge New Ones. Cell. 2014;157:77-94.

26. Vaucheret H. Post-transcriptional small RNA pathways in plants: Mechanisms and regulations. Genes Dev. 2006;20:759-71.

27. Axtell MJ. ShortStack: comprehensive annotation and quantification of small RNA genes. RNA. 2013:19:740-51.

28. Grant-Downton R, Rodriguez-Enriquez J. Emerging Roles for Non-Coding RNAs in Male Reproductive Development in Flowering Plants. Biomolecules. 2012;2:608-21

29. Zhang Y-C, Liao J-Y, Li Z-Y, Yu Y, Zhang J-P, Li Q-F, Qu L-H, Shu W-S, Chen $Y-Q$. Genome-wide screening and functional analysis identify a large number of long noncoding RNAs involved in the sexual reproduction of rice. Genome Biol. 2014;15:512.

30. Zhai J, Zhang H, Arikit S, Huang K, Nan G-L, Walbot V, Meyers BC. Spatiotemporally dynamic, cell-type-dependent premeiotic and meiotic phasiRNAs in maize anthers. Proc Natl Acad Sci. 2015;112:3146-51.

31. Axtell MJ. Non-coding RNAs: The small mysteries of males. Nat Plants. 2015;1:15055.

32. Jovanka A. Roles of Interspecific Hybridization and Cytogenetic Studies in Sunflower. Helia. 2004;27:1-24.

33. de la Paz J, Gómez M, Reyes-Valdés MH. Análisis meiótico de una cruza entre girasol cultivado (Helianthus annuus L. var. macrocarpus) y girasol silvestre (Helianthus annuus L. ssp. texanus Heiser). Acta Botánica Mex. 2007:80:7-20.

34. Ross-lbarra J. The evolution of recombination under domestication: a test of two hypotheses. Am Nat. 2004;163:105-12.

35. García-Ortega LF, Martínez O. How Many Genes Are Expressed in a Transcriptome? Estimation and Results for RNA-Seq. PLoS One. 2015;10, e0130262.

36. Kong L, Zhang Y, Ye Z-Q, Liu X-Q, Zhao S-Q, Wei L, Gao G. CPC: assess the protein-coding potential of transcripts using sequence features and support vector machine. Nucleic Acids Res. 2007:35(Web Server issue):W345-9.

37. Wang L, Park HJ, Dasari S, Wang S, Kocher J-P, Li W. CPAT: Coding-Potential Assessment Tool using an alignment-free logistic regression model. Nucleic Acids Res. 2013;41:e74.

38. Yoon J-H, Abdelmohsen K, Gorospe M. Posttranscriptional Gene Regulation by Long Noncoding RNA. J Mol Biol. 2013;425:3723-30.

39. Vitulo N, Forcato C, Carpinelli E, Telatin A, Campagna D, D'Angelo M, Zimbello R, Corso M, Vannozzi A, Bonghi C, Lucchin M, Valle G. A deep survey of alternative splicing in grape reveals changes in the splicing machinery related to tissue, stress condition and genotype. BMC Plant Biol. 2014;14:99.

40. Ding D-Q, Okamasa K, Yamane M, Tsutsumi C, Haraguchi T, Yamamoto M, Hiraoka Y. Meiosis-specific noncoding RNA mediates robust pairing of homologous chromosomes in meiosis. Science. 2012;336:732-6.

41. Ma J, Yan B, Qu Y, Qin F, Yang Y, Hao X, Yu J, Zhao Q, Zhu D, Ao G. Zm401, a short-open reading-frame mRNA or noncoding RNA, is essential for tapetum and microspore development and can regulate the floret formation in maize. J Cell Biochem. 2008;105:136-46.

42. Ding J, Lu Q, Ouyang Y. A long noncoding RNA regulates photoperiodsensitive male sterility, an essential component of hybrid rice. Proc Natl Acad Sci U S A. 2012:109:2654-9.

43. Soumillon $M$, Necsulea $A$, Weier $M$, Brawand $D$, Zhang $X, G u H$, Barthès $P$, Kokkinaki M, Nef S, Gnirke A, Dym M, de Massy B, Mikkelsen TS, Kaessmann $\mathrm{H}$. Cellular source and mechanisms of high transcriptome complexity in the mammalian testis. Cell Rep. 2013:3:2179-90.

44. Martínez O, Reyes-Valdés MH. Defining diversity, specialization, and gene specificity in transcriptomes through information theory. Proc Natl Acad Sci U S A. 2008;105:9709-14.

45. Necsulea A, Soumillon M, Warnefors M, Liechti A, Daish T, Zeller U, Baker JC, Grützner F, Kaessmann $\mathrm{H}$. The evolution of IncRNA repertoires and expression patterns in tetrapods. Nature. 2014;505:635-40.

46. Li L, Eichten SR, Shimizu R, Petsch K, Yeh C-T, Wu W, Chettoor AM, Givan SA, Cole RA, Fowler JE, Evans MMS, Scanlon MJ, Yu J, Schnable PS, Timmermans MCP, Springer NM, Muehlbauer GJ. Genome-wide discovery and characterization of maize long non-coding RNAs. Genome Biol. 2014;15:R40.

47. Komiya R, Ohyanagi H, Niihama M, Watanabe T, Nakano M, Kurata N, Nonomura K-I. Rice Germline-specific Argonaute MEL1 protein binds to phasiRNAs generated from more than 700 lincRNAs. Plant J. 2014;78:385-97.

48. Wu H-J, Wang Z-M, Wang M, Wang $X$-J. Widespread long noncoding RNAs as endogenous target mimics for microRNAs in plants. Plant Physiol. 2013;161:1875-84

49. Sharma V, Khurana S, Kubben $N$, Abdelmohsen $K$, Oberdoerffer $P$. Gorospe M, Misteli T. A BRCA 1 -interacting IncRNA regulates homologous recombination. EMBO Rep. 2015:16:1520-34.

50. Seeliger K, Dukowic-Schulze S, Wurz-Wildersinn R, Pacher M, Puchta H. BRCA2 is a mediator of RAD51- and DMC1-facilitated homologous recombination in Arabidopsis thaliana. New Phytol. 2012;193:364-75.

51. Zhang H, Chen X, Wang C, Xu Z, Wang Y, Liu X, Kang Z, Ji W. Long noncoding genes implicated in response to stripe rust pathogen stress in wheat (Triticum aestivum L.). Mol Biol Rep. 2013;40:6245-53.

52. Zhou Z-Y, Li A-M, Adeola AC, Liu Y-H, Irwin DM, Xie H-B, Zhang Y-P. Genome-wide identification of long intergenic noncoding RNA genes and their potential association with domestication in pigs. Genome Biol Evol. 2014;6:1387-92.

53. Swanson-Wagner R, Briskine R, Schaefer R, Hufford MB, Ross-lbarra J, Myers CL, Tiffin P, Springer NM. Reshaping of the maize transcriptome by domestication. Proc Natl Acad Sci U S A. 2012:109:11878-83.

54. Ma X, Shao C, Jin Y, Wang H, Meng Y. Long non-coding RNAs: A novel endogenous source for the generation of Dicer-like 1-dependent small RNAs in Arabidopsis thaliana. RNA Biol. 2014;11:373-90.

55. Shuai P, Liang D, Tang S, Zhang Z, Ye C-Y, Su Y, Xia X, Yin W. Genome-wide identification and functional prediction of novel and drought-responsive lincRNAs in Populus trichocarpa. J Exp Bot. 2014;65:4975-83.

56. Matzke M, Kanno T, Daxinger L, Huettel B, Matzke AJ. RNA-mediated chromatin-based silencing in plants. Curr Opin Cell Biol. 2009;21:367-76.

57. Mi S, Cai T, Hu Y, Chen Y, Hodges E, Ni F, Wu L, Li S, Zhou H, Long C, Chen S, Hannon GJ, Qi Y. Sorting of Small RNAs into Arabidopsis Argonaute Complexes is Directed by the 5' Terminal Nucleotide. Cell. 2008;133:116-27.

58. Vaucheret H. Plant ARGONAUTES. Trends Plant Sci. 2008;13:350-8.

59. Lelandais-Brière C, Sorin C, Declerck M, Benslimane A, Crespi M, Hartmann C. Small RNA diversity in plants and its impact in development. Curr Genomics. 2010;11:14-23.

60. Pantaleo V, Szittya G, Moxon S, Miozzi L, Moulton V, Dalmay T, Burgyan J. Identification of grapevine microRNAs and their targets using highthroughput sequencing and degradome analysis. Plant J. 2010;62:960-76.

61. Han R, Jian C, Lv J, Yan Y, Chi Q, Li Z, Wang Q, Zhang J, Liu X, Zhao H. Identification and characterization of microRNAs in the flag leaf and developing seed of wheat (Triticum aestivum L.). BMC Genomics. 2014;15:289. 
62. Montes RAC, de Fátima Rosas-Cárdenas F, De Paoli E, Accerbi M, Rymarquis LA, Mahalingam G, Marsch-Martínez N, Meyers BC, Green PJ, de Folter S. Sample sequencing of vascular plants demonstrates widespread conservation and divergence of microRNAs. Nat Commun. 2014;5.

63. Omidvar V, Mohorianu I, Dalmay T, Fellner M. Identification of miRNAs with potential roles in regulation of anther development and male-sterility in 7B-1 male-sterile tomato mutant. BMC Genomics. 2015;16:878.

64. Borges F, Pereira PA, Slotkin RK, Martienssen RA, Becker JD. MicroRNA activity in the Arabidopsis male germline. J Exp Bot. 2011;62:1611-20.

65. Wei M, Wei H, Wu M, Song M, Zhang J, Yu J, Fan S, Yu S. Comparative expression profiling of miRNA during anther development in genetic male sterile and wild type cotton. BMC Plant Biol. 2013;19:66.

66. Borsani O, Zhu J, Verslues PE, Sunkar R, Zhu J-K. Endogenous siRNAs Derived from a Pair of Natural cis-Antisense Transcripts Regulate Salt Tolerance in Arabidopsis. Cell. 2005:123:1279-91.

67. Chen D, Meng Y, Ma X, Mao C, Bai Y, Cao J, Gu H, Wu P, Chen M. Small RNAs in angiosperms: Sequence characteristics, distribution and generation. Bioinformatics. 2010;26:1391-4.

68. Johnson R, Guigó R. The RIDL hypothesis : transposable elements as functional domains of long noncoding RNAs. RNA. 2014;20:959-76.

69. Kannan S, Chernikova D, Rogozin IB, Poliakov E, Managadze D, Koonin EV, Milanesi L. Transposable Element Insertions in Long Intergenic Non-Coding RNA Genes. Front Bioeng Biotechnol. 2015;3(June):71.

70. Wang X, Ai G, Zhang C, Cui L, Wang J, Li H, Zhang J, Ye Z. Expression and diversification analysis reveals transposable elements play important roles in the origin of Lycopersicon-specific IncRNAs in tomato. New Phytol. 2015.

71. Natali L, Cossu R, Barghini E, Giordani T, Buti M, Mascagni F, Morgante M, Gill N, Kane NC, Rieseberg L, Cavallini A. The repetitive component of the sunflower genome as shown by different procedures for assembling next generation sequencing reads. BMC Genomics. 2013;14:686.

72. Giordani T, Cavallini A, Natali L. The repetitive component of the sunflower genome. Curr Plant Biol. 2014;1:45-54

73. Olsen KM, Wendel JF. A Bountiful Harvest: Genomic Insights into Crop Domestication Phenotypes. Annu Rev Plant Biol. 2013;64:47-70.

74. Slotkin RK, Martienssen R. Transposable elements and the epigenetic regulation of the genome. Nat Rev Genet. 2007:8:272-85.

75. Olmedo-Monfil V, Durán-Figueroa N, Arteaga-Vázquez M, Demesa-Arévalo $E$ Autran D, Grimanelli D, Slotkin RK, Martienssen RA, Vielle-Calzada J-P. Control of female gamete formation by a small RNA pathway in Arabidopsis. Nature. 2010;464:628-32.

76. Li J, Ma W, Zeng P, Wang J, Geng B, Yang J, Cui Q. LncTar: A tool for predicting the RNA targets of long noncoding RNAs. Brief Bioinform. 2014;16:806-12.

77. Dawe RK, Sedat JW, Agard DA, Cande WZ. Meiotic chromosome pairing in maize is associated with a novel chromatin organization. Cell. 1994;76:901-12.

78. Oliver C, Pradillo M, Corredor E, Cuñado N. The dynamics of histone H3 modifications is species-specific in plant meiosis. Planta. 2013;238:23-33.

79. Amaral PP, Dinger ME, Mercer TR, Mattick JS. The eukaryotic genome as an RNA machine. Science. 2008;319:1787-9.

80. Derrien T, Johnson R, Bussotti G, Tanzer A, Djebali S, Tilgner H, Guernec G, Martin D, Merkel A, Knowles DG, Lagarde J, Veeravalli L, Ruan X, Ruan Y, Lassmann T, Carninci P, Brown JB, Lipovich L, Gonzalez JM, Thomas M, Davis CA, Shiekhattar R, Gingeras TR, Hubbard TJ, Notredame C, Harrow J, Guigó R. The GENCODE v7 catalog of human long noncoding RNAs: analysis of their gene structure, evolution, and expression. Genome Res. 2012;22:1775-89.

81. Stages of Sunflower Development. doi:10.2135/cropsci1981. $0011183 \times 002100060024 x$

82. Martin M. Cutadapt removes adapter sequences from high-throughput sequencing reads. EMBnet journal. 2011;17:10.

83. Schmieder R, Edwards R. Quality control and preprocessing of metagenomic datasets. Bioinformatics. 2011;27:863-4.

84. Grabherr MG, Haas BJ, Yassour M, Levin JZ, Thompson DA, Amit I, Adiconis X, Fan L, Raychowdhury R, Zeng Q, Chen Z, Mauceli E, Hacohen N, Gnirke A, Rhind N, di Palma F, Birren BW, Nusbaum C, Lindblad-Toh K, Friedman N, Regev A. Full-length transcriptome assembly from RNA-Seq data without a reference genome. Nat Biotechnol. 2011;29:644-52.

85. Langmead B, Salzberg SL. Fast gapped-read alignment with Bowtie 2. Nat Methods. 2012;9:357-9.
86. Roberts A, Pachter L. Streaming fragment assignment for real-time analysis of sequencing experiments. Nat Methods. 2013;10:71-3.

87. Altschul SF, Madden TL, Schäffer AA, Zhang J, Zhang Z, Miller W, Lipman DJ. Gapped BLAST and PSI-BLAST: a new generation of protein database search programs. Nucleic Acids Res. 1997;25:3389-402.

88. Davis MP A, van Dongen S, Abreu-Goodger C, Bartonicek N, Enright AJ. Kraken: A set of tools for quality control and analysis of high-throughput sequence data. Methods. 2013;63:41-9.

89. Langmead B, Trapnell C, Pop M, Salzberg SL. Ultrafast and memory-efficient alignment of short DNA sequences to the human genome. Genome Biol. 2009;10:R25.

90. Chen D, Yuan C, Zhang J, Zhang Z, Bai L, Meng Y, Chen L-L, Chen M. PlantNATsDB: a comprehensive database of plant natural antisense transcripts. Nucleic Acids Res. 2012;40:1187-93.

91. Jurka J. Repbase Update: A database and an electronic journal of repetitive elements. Trends Genet. 2000;16:418-20.

92. Nussbaumer T, Martis MM, Roessner SK, Pfeifer M, Bader KC, Sharma S, Gundlach H, Spannagl M. MIPS PlantsDB: A database framework for comparative plant genome research. Nucleic Acids Res. 2013;41:1144-51.

93. Robinson MD, McCarthy DJ, Smyth GK. edgeR: a Bioconductor package for differential expression analysis of digital gene expression data. Bioinformatics. 2010:26:139-40.

94. Storey JD, Tibshirani R. Statistical significance for genomewide studies. Proc Natl Acad Sci U S A. 2003;100:9440-5.

95. R Core Team. R: A Language and Environment for Statistical Computing. R Foundation for Statistical Computing, Vienna, Austria. 2013.

\section{Submit your next manuscript to BioMed Central and we will help you at every step:}

- We accept pre-submission inquiries

- Our selector tool helps you to find the most relevant journal

- We provide round the clock customer support

- Convenient online submission

- Thorough peer review

- Inclusion in PubMed and all major indexing services

- Maximum visibility for your research

Submit your manuscript at www.biomedcentral.com/submit
Biomed Central 